\title{
Le dépistaǵe du cancer colorectal au Canada : Résultats d'une enquête nationale
}

\author{
M.J. Sewitch, Ph.D. (1); C. Fournier, M.Sc. (2); A. Ciampi, Ph.D. (3); A. Dyachenko, M.Sc. (4)
}

\section{Résumé}

Les lignes directrices canadiennes recommandent le dépistage du cancer colorectal (CCR) pour les personnes âgées de 50 à 74 ans. L'étude vise à estimer les taux du dépistage du CCR selon des caractéristiques individuelles et géographiques et de l'observation des lignes directrices en vigueur à l'égard du dépistage du CCR. Des enquêtés du cycle 2.1 de l'Enquête sur la santé dans les collectivités canadiennes 2003 (âgés de $\geq 50$ ans, sans CCR passé ou présent) ont participé à l'étude. Les taux d'utilisation et de dépistage par recherche de sang occulte dans les selles (RSOS) et endoscopie ont été calculés. L'échantillon comprend 16747 résidents de Terre-Neuve, de l'Ontario, de la Saskatchewan et de la ColombieBritannique. Dans l'ensemble, le taux de dépistage par RSOS était de $7,7 \%$ au cours de la dernière année et le taux de dépistage par endoscopie était de 8,8 \% dans les cinq dernières années. Les taux de dépistage par RSOS sont plus élevés chez les personnes sondées plus âgées et les personnes sondées de sexe masculin; les taux de dépistage par endoscopie sont plus élevés chez les interrogés plus âgés. Les personnes de 50 à 59 ans et de plus de 90 ans étaient les moins susceptibles d'avoir subi un dépistage. Environ 70 \% des personnes sondées n’observaient pas les lignes directrices en vigueur sur le dépistage $d u$ CCR. Les taux d'inobservation étaient plus élevés dans la plupart des régions sanitaires de la Colombie-Britannique. Les données de l'enquête nationale semblent indiquer que le dépistage du CCR au Canada est faible; les personnes plus jeunes et les résidents de la Colombie-Britannique sont les moins susceptibles d'avoir subi un test de dépistage du CCR.

Mots clés : cancer colorectal, dépistage, enquête, RSOS, endoscopie

\section{Introduction}

Au Canada, le cancer colorectal (CCR) est le quatrième cancer le plus diagnostiqué et la deuxième et troisième principale cause des décès dus au cancer chez les hommes et les femmes, respectivement ${ }^{1}$. Le dépistage du CCR réduit tant l'incidence du CCR grâce à l'ablation de polypes précancéreux que les décès dus au CCR grâce à la détection et au traitement précoces. Depuis 1996, plusieurs organismes ont publié des lignes directrices relatives au dépistage du CCR pour les personnes à risque moyen, soit les personnes de 50 ans et plus présentant un risque moyen de
CCR. Les lignes directrices canadiennes recommandent un test de dépistage par recherche de sang occulte dans les selles (RSOS) (un à deux ans ${ }^{2-4}$ ) tandis que les lignes directrices des États-Unis recommandent une fréquence annuelle ${ }^{5,6}$. Pour d'autres modalités de dépistage, le Canada et les États-Unis recommandent des fréquences identiques : aux dix ans pour la colonoscopie et aux cinq ans pour la sigmoïdoscopie flexible et le lavement baryté à double contraste, bien que les lignes directrices des États-Unis recommandent aussi une combinaison de la RSOS annuelle et de la sigmoïdoscopie flexible aux cinq ans.
Malgré la diffusion généralisée des lignes directrices relatives au dépistage du CCR, il demeure sous-utilisé. Aux États-Unis, plusieurs études ont employé des données d'enquêtes nationales et collecté des données par composition aléatoire de numéros de téléphone ou au moyen d'entrevues individuelles. Des études fondées sur les données du National Health Interview Survey ${ }^{7}$, du Behavioral Risk Factor Surveillance System ${ }^{8}$, du Community Quality Index ${ }^{9}$, du California Health Interview Survey ${ }^{10}$ et du Health Information National Trends Survey ${ }^{11}$ ont révélé que les taux de dépistage du CCR variaient de $15 \%$ à $65 \%$ selon la période à l'étude. En Ontario, des études fondées soit sur des données administratives ou des données d'enquête estiment que moins de $25 \%$ de la population admissible au dépistage s'est soumise à un test de dépistage $\mathrm{e}^{12-14}$. En Alberta, une enquête basée sur une population a révélé que 14,3\% seulement des personnes présentant un risque moyen étaient réputées à jour en matière de dépistage du $\mathrm{CCR}^{15}$.

Au moment où le dépistage du CCR passe à l'avant-plan des soins de santé préventifs grâce à une sensibilisation du grand public et des professionnels, les taux de dépistage du CCR au Canada revêtent un intérêt grandissant. Toutefois, la mesure dans laquelle les Canadiens subissent des tests de dépistage du CCR en accord avec les lignes directrices demeure inconnue. De plus, nous en savons peu sur les caractéristiques des personnes qui subissent un dépistage du CCR et sur l'utilisation des procédures de dépistage du CCR au fil du temps. Vu le lancement de plusieurs

Coordonnées des auteurs

1 Faculté de médecine, Université McGill, Montréal (Québec)

2 Division de l'épidémiologie clinique, Institut de recherche du Centre universitaire de santé McGill, Montréal (Québec)

3 Département d'épidémiologie et de biostatistique, Université McGill, Montréal (Québec)

4 Département d'épidémiologie et d'études communautaires, Centre hospitalier St. Mary's, Montréal (Québec)

Correspondance : Maida J. Sewitch, Ph.D., 687 avenue Pine Ouest, Édifice V, Hôpital Royal Victoria, Montréal (Québec) H3A 1A1, Tél. : 514-934-1934 poste 44736, Téléc. : 514-934-8293,

Courriel : maida.sewitch@mcgill.ca 
programmes provinciaux de dépistage du CCR au Canada, il est essentiel de saisir les écarts en fait de dépistage du CCR pour favoriser la planification, la mise en œuvre et le fonctionnement efficaces des initiatives de dépistage du CCR. Par conséquent, la présente étude basée sur une population vise à estimer les taux d'utilisation 1) de la RSOS et de l'endoscopie en guise de procédures de dépistage du CCR et 2) l'observation des lignes directrices en vigueur relatives au dépistage du CCR.

\section{Méthodes}

\section{Sources de données}

La principale source de données est l'Enquête sur la santé dans les collectivités canadiennes (ESCC), cycle 2.1 (janvier à décembre 2003) de Statistique Canada ${ }^{16}$, qui vise à fournir des estimations des déterminants de la santé, de l'état de santé et de l'utilisation du système de santé au Canada. Cette enquête porte sur les résidents des ménages âgés de 12 ans et plus dans l'ensemble des provinces et des territoires, exception faite des personnes vivant dans des réserves indiennes ou dans des régions éloignées ainsi que des membres à temps plein des Forces armées canadiennes. Le cycle 2.1 de l'ESCC fournit des renseignements sociodémographiques de base sur tous les enquêtés dans toutes les régions sanitaires, mais le module sur le dépistage du CCR était administré à la discrétion des régions sanitaires. Les enquêtés au module sur le dépistage du CCR proviennent de toutes les régions sanitaires de Terre-Neuve et de la ColombieBritannique et, respectivement, de 14 sur 37 et de 7 sur 11 régions sanitaires de l'Ontario et de la Saskatchewan ${ }^{17}$. Les régions sanitaires sont définies par les ministères de la Santé provinciaux et se composent généralement de régions administratives définies par des lois, représentant des territoires géographiques sous la responsabilité de conseils hospitaliers ou d'autorités sanitaires régionales ${ }^{18}$. Les données d'enquête sont non nominatives. Les interrogés ne sont pas identifiés.

\section{Population de l'étude}

La population de l'étude comprend les interrogés à l'enquête qui ont rempli le module de dépistage du CCR et déclaré avoir 50 ans et plus, sans CCR passé ou présent. Les interrogés n'ayant pas fourni d'information sur la dernière utilisation déclarée de la RSOS ont été exclus. Les caractéristiques sociodémographiques comprennent l'âge, le sexe, le plus haut niveau de scolarité atteint et le revenu du ménage. Les caractéristiques cliniques comprennent la maladie entérique (avoir reçu d'un professionnel de la santé un diagnostic de maladie de Crohn ou de colite ulcéreuse). Les caractéristiques géographiques comprennent la région résidentielle (urbaine par opposition à rurale), la région sanitaire et la province de résidence.

\section{Variables des résultats}

Les taux de dépistage par RSOS et endoscopie (c.-à-d., sigmoïdoscopie ou colonoscopie) et l'utilisation de ces procédures sont fondés sur les réponses au questionnaire servant à évaluer la dernière fois que la procédure a été utilisée ainsi que ce qui y a été déclaré, c.-à-d., (dépistage ou nondépistage). Les taux de dépistage proviennent des procédures exécutées selon «l'examen régulier », «l'âge », « la race » ou " les antécédents familiaux de CCR ». Le lien de parenté avec le membre de la famille touché n’a pas été évalué. Les taux d'utilisation comprennent les procédures de dépistage et les procédures exécutées pour " suivi d'un problème antérieur » ou « autres raisons » (non-dépistage). La RSOS sert également dans un cadre autre que celui de dépistage, par exemple, pour déceler la présence de sang dans les selles d'un patient anémié. Trois taux d'observation sont définis en fonction des périodicités recommandées dans les lignes directrices relatives au dépistage du CCR en vigueur au moment de l'étude. (1) L'observation des lignes directrices relatives au dépistage par RSOS est définie comme le fait d'avoir subi une RSOS au cours des deux dernières années; (2) l'observation des lignes directrices relatives au dépistage par endoscopie est définie comme le fait d'avoir subi une endoscopie au cours des dix dernières années; (3) l'observation des lignes directrices en vigueur relatives au dépistage du CCR est définie en fonction du classement soit dans (1) soit dans (2). Les trois taux d'observation, fondés sur les procédures exécutées pour toutes les indications, fournissent une estimation du nombre d'interrogés réputés à jour en matière de dépistage du CCR. L'hypothèse sousjacente, c'est qu'une fois que la procédure est exécutée, la répétition du test à des fins de dépistage devrait suivre les périodicités recommandées dans les lignes directrices.

\section{Analyse statistique}

Des statistiques descriptives permettent de caractériser la population de l'étude dans son ensemble et selon la modalité de dépistage. Les taux de dépistage sont calculés selon le nombre de personnes sondées déclarant avoir subi une procédure de dépistage, divisé par ceux qui ont déclaré avoir subi une procédure de dépistage plus ceux déclarant ne jamais avoir subi de procédure (et le tout dans les intervalles de temps établis). Les interrogés qui ont subi la procédure à d'autres fins que le dépistage sont exclus. Nous avons calculé les taux d'utilisation selon le nombre de personnes sondées déclarant avoir subi une procédure pour quelque indication que ce soit (dépistage, non-dépistage) divisé par le nombre total de personnes sondées dans la population à l'étude. Nous avons calculé les taux en fonction de la dernière utilisation déclarée de la procédure. Les taux de RSOS sont calculés en fonction des cinq intervalles de temps suivants : moins d'un an, un à deux ans, deux à trois ans, plus de trois ans et jamais. Nous avons calculé les taux d'endoscopie en fonction des quatre intervalles de temps suivants : dans les cinq dernières années, de six à dix ans, plus de dix ans et jamais. Ces intervalles de temps diffèrent des périodicités recommandées dans les lignes directrices pour permettre d'établir des comparaisons au fil du temps. Nous avons calculé les taux de dépistage et d'utilisation globaux pour l'ensemble de la population à l'étude. Nous avons aussi calculé les taux de dépistage selon le sexe, le groupe d'âge, le niveau de revenu du ménage, le niveau de scolarité, l'état de santé par rapport aux maladies entériques et les régions géographiques (région résidentielle, région sanitaire et province de résidence).

Nous avons calculé les taux en agrégeant des données pondérées pour les régions sanitaires participantes. Les taux peuvent ne pas être représentatifs de l'ensemble de la province si quelques régions sanitaires 
seulement sont échantillonnées. Par conséquent, nous ne rapportons que des taux de dépistage régionaux pour l'Ontario et la Saskatchewan. Par contre, nous rapportons des taux de dépistage régionaux et provinciaux pour Terre-Neuve et la Colombie-Britannique puisque toutes les régions sanitaires ont été échantillonnées. Nous avons calculé les trois résultats en fait d'observation de façon globale et par région sanitaire. Les taux d'observation des lignes directrices en vigueur relatives au dépistage du CCR sont fondés sur l'utilisation de la RSOS et de l'endoscopie, mais nous avons inclus les enquêtés ayant fourni des renseignements valides au sujet d'une procédure seulement et nous les avons classés en conséquence. Nous avons employé les pondérations d'autoamorçage de Statistique Canada pour calculer l'intervalle de confiance de $95 \%$ (IC $95 \%$ ) à l'aide du programme BOOTWARE_V30 (version 3.0) ${ }^{19}$. Toutes les analyses ont été exécutées à l'aide du logiciel statistique $\mathrm{SAS}^{20}$.

Nous avons estimé la taille de la population canadienne à l'époque de l'enquête et de la population de l'étude (c.-à-d. le nombre de Canadiens représentés par les interrogés) au moyen des pondérations susmentionnées. Tous les chiffres présentés sont des valeurs pondérées, en accord avec les politiques de Statistique Canada.

\section{Résultats}

\section{Population de l'étude}

Le module sur le dépistage du CCR a été administré à 39178 personnes (figure 1). De ce nombre, 16747 enquêtés remplissaient les critères d'admissibilité et on a estimé qu'ils représentaient 2394124 Canadiens (selon les procédures de pondération). On estime la taille de la population canadienne âgée de 12 ans et plus à 26578128 personnes. Parmi les enquêtés admissibles, 16745 et 16648 ont fourni des renseignements sur l'utilisation de la RSOS et de l'endoscopie, respectivement; 14482 et 13949 ont fourni des renseignements sur le dépistage au moyen de la RSOS et de l'endoscopie, respectivement.

Le tableau 1 renferme les caractéristiques sociodémographiques, cliniques et géographiques de la population de l'étude dans l'ensemble et selon la modalité de dépistage. Dans l'ensemble, les sous-groupes les plus nombreux sont les femmes, les personnes de 50 à 64 ans, ceux qui ont fait des études postsecondaires, les enquêtés nés au Canada, de race blanche, sans emploi, sans maladie entérique, vivant en région urbaine et en Colombie-Britannique. Parmi les 14482 enquêtés ayant fourni des renseignements sur le dépistage par RSOS, $21,6 \%$ en ont subi un au cours de leur vie. Comparativement à la population totale de l'étude, un plus fort pourcentage des personnes déclarant avoir subi un dépistage par RSOS sont âgées de 50 à 64 ans et n'ont pas d'emploi à l'extérieur de la maison. Parmi les 13949 enquêtés ayant fourni des renseignements sur le dépistage par endoscopie, 11,3\% ont subi cette forme de dépistage au cours de leur vie. Comparativement à la population totale de l'étude, un plus fort pourcentage des personnes déclarant avoir subi un dépistage

FIGURE 1

Sélection de la population de l'étude parmi les interrogés au module sur le dépistage du CCR du cycle 2.1 de l'ESCC

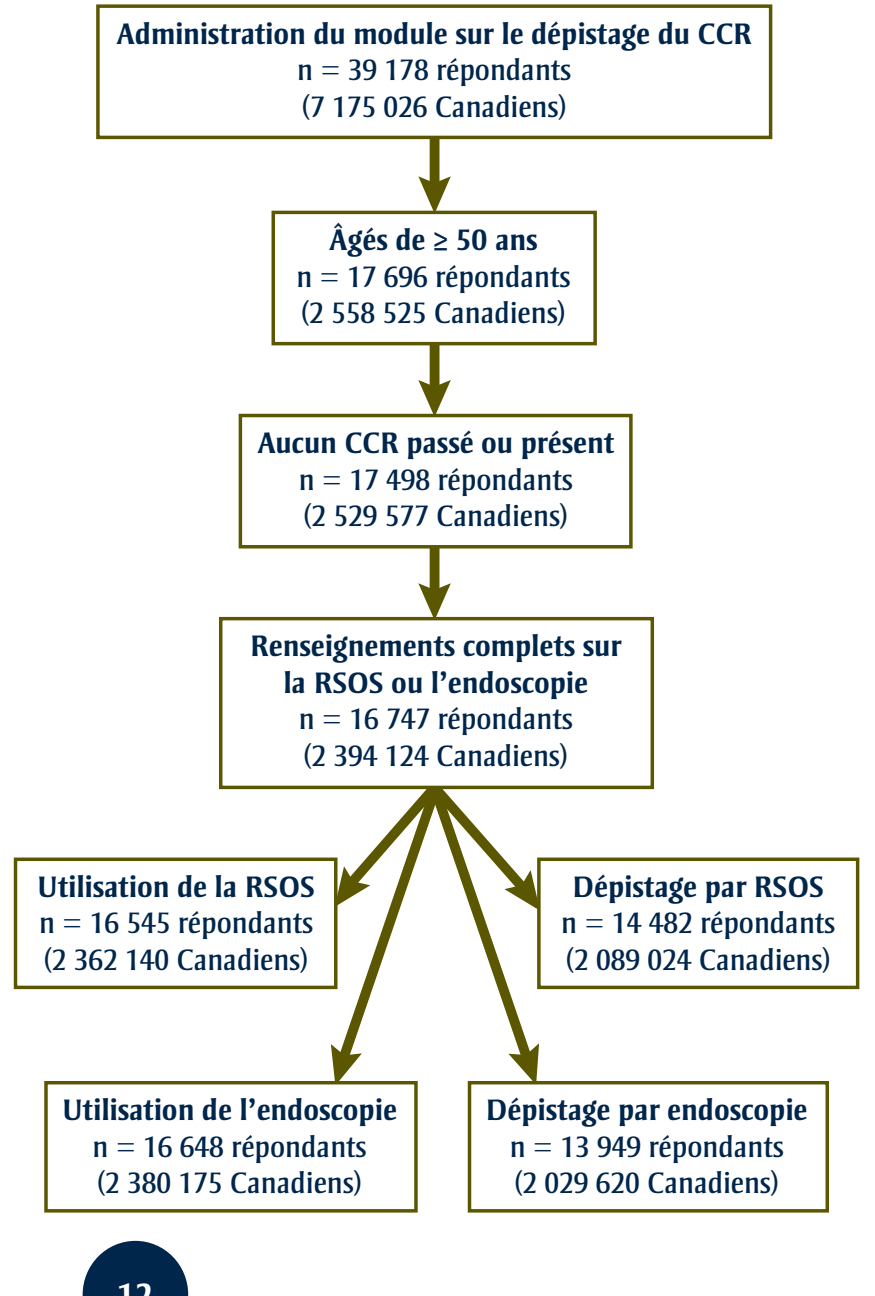

par endoscopie n’ont pas d'emploi à l'extérieur de la maison et résidaient en Ontario; une proportion plus petite d'entre eux habitent la Colombie-Britannique.

\section{Taux de dépistage par RSOS}

Le tableau 2 présente les taux de dépistage par RSOS selon l'intervalle de temps et des caractéristiques sociodémographiques, cliniques et géographiques. Dans l'ensemble, les taux de dépistage par RSOS étaient de $7,7 \%$ au cours de l'année précédente, de 5,1\% entre un et deux ans auparavant, $2,5 \%$ entre deux et trois ans auparavant et $6,3 \%$ il y a trois ans et plus; 78,4\% des personnes sondées n'ont jamais subi de dépistage par RSOS. Les taux de dépistage par RSOS dans l'année précédente et un à deux ans auparavant sont plus élevés chez les hommes, les interrogés ayant une maladie entérite et les interrogés de 65 ans et plus. Les taux sont les plus faibles dans le groupe des 50 ans à 59 ans. Aucune 
TABLEAU 1

Caractéristiques sociodémographiques, cliniques et géographiques de la population de l'étude selon le statut de dépistage par RSOS et endoscopie

\begin{tabular}{|c|c|c|c|c|c|c|c|}
\hline \multirow[t]{2}{*}{ Caractéristique } & \multirow[t]{2}{*}{ Catégorie } & \multirow{2}{*}{$\begin{array}{c}\text { Global } \\
\text { n (2 } 394 \text { 124) }\end{array}$} & \multicolumn{3}{|c|}{ Dépistage par RSOSa } & \multicolumn{2}{|c|}{$\begin{array}{l}\text { Dépistage par } \\
\text { endoscopie }^{\mathrm{b}} w\end{array}$} \\
\hline & & & $\%^{c}$ & n (451 669) & $\%^{c}$ & n (229 578) & $\%^{c}$ \\
\hline \multicolumn{8}{|l|}{ Sociodémographique } \\
\hline \multirow[t]{2}{*}{ Sexe } & Masculin & 1140566 & 47,6 & 233372 & 51,7 & 112541 & 49,0 \\
\hline & Féminin & 1253559 & 52,4 & 218298 & 48,3 & 117037 & 51,0 \\
\hline \multirow[t]{2}{*}{ Âge } & 50 à 64 ans & 1429679 & 59,7 & 222473 & 49,3 & 122450 & 53,3 \\
\hline & 65 ans et plus & 964446 & 40,3 & 229197 & 50,7 & 107128 & 46,7 \\
\hline \multirow[t]{4}{*}{ Études } & $<$ Niveau secondaire & 630647 & 27,0 & 110676 & 25,1 & 56249 & 25,0 \\
\hline & $\begin{array}{l}\text { Diplôme d'études } \\
\text { secondaires. }\end{array}$ & 462201 & 19,8 & 89306 & 20,3 & 42533 & 18,9 \\
\hline & Postsecondaire & 143139 & 6,1 & 23708 & 5,4 & 17793 & 7,9 \\
\hline & $\begin{array}{l}\text { Diplôme d'études } \\
\text { postsecondaires }\end{array}$ & 1095953 & 47,0 & 216554 & 49,2 & 108544 & 48,2 \\
\hline \multirow[t]{2}{*}{ Pays de naissance } & Canada & 1653452 & 70,3 & 315685 & 70,9 & 171337 & 75,5 \\
\hline & Autres & 697280 & 29,7 & 129554 & 29,1 & 55543 & 24,5 \\
\hline \multirow[t]{2}{*}{ Origine culturelle / raciale } & Race blanche & 2067992 & 88,0 & 405037 & 91,1 & 205344 & 90,8 \\
\hline & Autres & 281342 & 12,0 & 39630 & 8,9 & 20748 & 9,2 \\
\hline \multirow[t]{3}{*}{ Revenu du ménage } & Faible à moyen inférieur & 656704 & 33,2 & 116925 & 31,1 & 64074 & 33,7 \\
\hline & Moyen supérieur & 685018 & 34,7 & 132543 & 35,2 & 64003 & 33,6 \\
\hline & Élevé & 635229 & 32,1 & 126742 & 33,7 & 62152 & 32,7 \\
\hline \multirow{3}{*}{$\begin{array}{l}\text { Statut d'emploi (au cours } \\
\text { de la dernière année) }\end{array}$} & Temps plein & 864382 & 37,0 & 126254 & 28,6 & 68193 & 30,2 \\
\hline & Temps partiel & 211809 & 9,1 & 39937 & 9,0 & 21600 & 9,6 \\
\hline & Sans emploi & 1258634 & 53,9 & 275747 & 62,4 & 135673 & 60,2 \\
\hline \multicolumn{8}{|l|}{ Clinique } \\
\hline \multirow[t]{2}{*}{ Maladie entérique } & Oui & 86080 & 3,6 & 16664 & 3,7 & 10699 & 4,7 \\
\hline & Non & 2305488 & 96,4 & 434428 & 96,3 & 218770 & 95,3 \\
\hline \multicolumn{8}{|l|}{ Géographique } \\
\hline \multirow[t]{2}{*}{ Région de résidence } & Urbaine & 1895462 & 79,2 & 355944 & 78,8 & 183465 & 79,9 \\
\hline & Rurale & 498663 & 20,8 & 95725 & 21,2 & 46114 & 20,1 \\
\hline \multirow[t]{4}{*}{ Province et résidence } & Terre-Neuve et Labrador & 155166 & 6,5 & 19859 & 4,4 & 15673 & 6,8 \\
\hline & Ontario & 889608 & 37,2 & 171566 & 38,0 & 111762 & 48,7 \\
\hline & Saskatchewan & 168279 & 7,0 & 31603 & 7,0 & 15508 & 6,8 \\
\hline & Colombie-Britannique & 1181072 & 49,3 & 228641 & 50,6 & 86636 & 37,7 \\
\hline
\end{tabular}

Tous les chiffres sont pondérés.

Les sommes des chiffres peuvent ne pas correspondre au $\mathrm{n}$ de la population à cause de données manquantes.

${ }^{a}$ Enquêtés déclarant avoir subi un dépistage par RSOS au cours de leur vie.

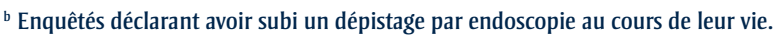

c Basé sur les réponses valides (exclut les valeurs manquantes). 
TABLEAU 2

Taux de dépistage par RSOS selon l'intervalle de temps en fonction des caractéristiques sociodémographiques, cliniques et géographiques $\left(n^{\mathrm{a}}=14482\right)$

\begin{tabular}{|c|c|c|c|c|c|c|c|c|c|c|c|c|c|c|c|}
\hline \multirow{3}{*}{$\begin{array}{l}\text { Caractéristique } \\
\text { GLOBAL }\end{array}$} & \multicolumn{15}{|c|}{ Dernier dépistage par RSOS déclaré } \\
\hline & \multirow{2}{*}{$\begin{array}{l}<1 \text { an } \\
\text { Taux } \\
7,7\end{array}$} & \multicolumn{2}{|c|}{ IC $95 \%$} & \multirow{2}{*}{$\begin{array}{l}1 \text { à } 2 \\
\text { Taux } \\
5,1\end{array}$} & \multicolumn{2}{|c|}{ IC $95 \%$} & \multicolumn{3}{|c|}{2 à 3 ans } & \multicolumn{3}{|c|}{3 ans et plus } & \multicolumn{3}{|c|}{$\begin{array}{l}\text { Jamais } \\
\text { Taux IC } 95 \%\end{array}$} \\
\hline & & 7,1 & 8,4 & & 4,6 & 5,6 & 2,5 & 2,2 & 2,8 & 6,3 & 5,8 & 6,9 & 78,4 & 77,5 & 79,3 \\
\hline \multicolumn{16}{|l|}{ Sexe } \\
\hline Masculin & 9,3 & 8,2 & 10,3 & 5,8 & 5,0 & 6,6 & 2,3 & 1,8 & 2,7 & 5,7 & 5,0 & 6,5 & 76,9 & 75,5 & 78,4 \\
\hline Féminin & 6,2 & 5,5 & 7,0 & 4,4 & 3,8 & 5,0 & 2,7 & 2,3 & 3,2 & 6,9 & 6,2 & 7,6 & 79,7 & 78,6 & 80,9 \\
\hline \multicolumn{16}{|l|}{ Âge (ans) } \\
\hline 50 à 64 & 6,6 & 5,7 & 7,4 & 4,5 & 3,8 & 5,1 & 1,8 & 1,5 & 2,2 & 4,8 & 4,1 & 5,4 & 82,4 & 81,2 & 83,6 \\
\hline 65 et plus & 9,5 & 8,4 & 10,6 & 6,0 & 5,3 & 6,8 & 3,5 & 3,0 & 4,1 & 8,7 & 7,9 & 9,6 & 72,2 & 70,7 & 73,7 \\
\hline 50 à 59 & 5,7 & 4,8 & 6,6 & 3,8 & 3,2 & 4,5 & 1,7 & 1,2 & 2,2 & 4,2 & 3,5 & 4,9 & 84,5 & 83,2 & 85,9 \\
\hline 60 à 69 & 9,3 & 8,1 & 10,5 & 5,9 & 4,9 & 6,9 & 2,7 & 2,1 & 3,3 & 6,5 & 5,4 & 7,6 & 75,6 & 73,8 & 77,4 \\
\hline 70 à 79 & 10,3 & 8,8 & 11,8 & 6,5 & 5,4 & 7,7 & 3,7 & 2,8 & 4,5 & 9,0 & 7,7 & 10,2 & 70,6 & 68,4 & 72,8 \\
\hline 80 à 89 & 7,7 & 4,7 & 10,6 & 6,2 & 4,5 & 8,0 & 3,8 & 2,7 & 5,0 & 11,8 & 9,5 & 14,2 & 70,5 & 66,8 & 74,1 \\
\hline 90 à 100 & $\mathrm{n} / \mathrm{a}$ & $\mathrm{n} / \mathrm{a}$ & $\mathrm{n} / \mathrm{a}$ & 3,7 & 0,6 & 6,8 & $\mathrm{n} / \mathrm{a}$ & $\mathrm{n} / \mathrm{a}$ & $\mathrm{n} / \mathrm{a}$ & 10,5 & $\mathrm{n} / \mathrm{a}$ & 21,5 & 83,0 & 72,0 & 93,9 \\
\hline \multicolumn{16}{|l|}{ Région de résidence } \\
\hline Urbaine & 7,7 & 6,9 & 8,4 & 4,9 & 4,4 & 5,4 & 2,6 & 2,2 & 3,0 & 6,4 & 5,8 & 7,0 & 78,5 & 77,4 & 79,5 \\
\hline Rurale & 7,9 & 6,7 & 9,2 & 5,8 & 4,7 & 6,8 & 2,0 & 1,5 & 2,6 & 6,3 & 5,3 & 7,3 & 78,0 & 76,1 & 79,8 \\
\hline \multicolumn{16}{|l|}{ Études } \\
\hline$<$ Niveau secondaire & 6,9 & 5,9 & 8,0 & 4,8 & 4,0 & 5,7 & 2,5 & 2,0 & 3,1 & 6,3 & 5,3 & 7,2 & 79,5 & 77,8 & 81,1 \\
\hline $\begin{array}{l}\text { Diplôme d'études } \\
\text { secondaires }\end{array}$ & 7,7 & 6,1 & 9,3 & 5,6 & 4,5 & 6,7 & 2,7 & 1,9 & 3,4 & 5,7 & 4,7 & 6,6 & 78,4 & 76,3 & 80,6 \\
\hline Études postsecondaires & 9,1 & 6,4 & 11,9 & 3,7 & 1,8 & 5,6 & 1,9 & 1,0 & 2,9 & 5,2 & 3,1 & 7,2 & 80,1 & 75,8 & 84,4 \\
\hline $\begin{array}{l}\text { Diplôme d'études } \\
\text { postsecondaires }\end{array}$ & 8,0 & 7,0 & 9,0 & 5,1 & 4,4 & 5,8 & 2,5 & 2,0 & 3,0 & 6,9 & 6,0 & 7,7 & 77,5 & 76,1 & 79,0 \\
\hline \multicolumn{16}{|l|}{ Revenu du ménage } \\
\hline Faible à moyen inférieur & 6,8 & 5,6 & 7,9 & 4,4 & 3,7 & 5,1 & 2,6 & 2,0 & 3,2 & 7,1 & 6,1 & 8,2 & 79,1 & 77,5 & 80,8 \\
\hline Moyen supérieur & 7,9 & 6,8 & 9,1 & 4,9 & 4,1 & 5,7 & 2,1 & 1,6 & 2,6 & 7,3 & 6,3 & 8,3 & 77,8 & 76,1 & 79,5 \\
\hline Élevé & 9,0 & 7,5 & 10,5 & 5,7 & 4,7 & 6,8 & 2,8 & 2,0 & 3,5 & 5,0 & 4,0 & 5,9 & 77,6 & 75,4 & 79,7 \\
\hline \multicolumn{16}{|l|}{ Maladie entérique } \\
\hline Oui & 11,9 & 7,8 & 15,9 & 5,5 & 2,8 & 8,2 & 3,0 & 1,2 & 4,8 & 10,1 & 6,5 & 13,8 & 69,5 & 63,8 & 75,1 \\
\hline Non & 7,6 & 6,9 & 8,2 & 5,1 & 4,5 & 5,6 & 2,5 & 2,2 & 2,8 & 6,2 & 5,7 & 6,8 & 78,6 & 77,7 & 79,6 \\
\hline \multicolumn{16}{|l|}{ Province de résidence $^{c}$} \\
\hline $\begin{array}{l}\text { Terre-Neuve } \\
\text { et Labrador }\end{array}$ & 3,8 & 2,7 & 4,9 & 2,8 & 1,7 & 3,8 & 2,0 & 0,8 & 3,1 & 6,1 & 4,6 & 7,7 & 85,4 & 83,2 & 87,5 \\
\hline Colombie-Britannique & 8,1 & 7,0 & 9,2 & 5,1 & 4,3 & 5,9 & 2,1 & 1,7 & 2,5 & 6,4 & 5,6 & 7,3 & 78,4 & 76,8 & 79,9 \\
\hline
\end{tabular}

Les pourcentages des taux représentent des données pondérées.

${ }^{a}$ Nombre de personnes sondées ayant fourni des renseignements sur le dépistage par RSOS et représentant 2089024 Canadiens.

${ }^{\mathrm{b}}$ Enquêtés classés selon la date de déclaration du dernier dépistage par RSOS.

Les RSOS exécutées à d'autres fins que le dépistage sont exclues.

c Les taux provinciaux pour l'Ontario et la Saskatchewan ne sont pas indiqués parce que nous ne disposons pas de données pour toutes les régions sanitaires.

n.d.= non disponible parce que les cellules de données non pondérées contenaient moins de 5 personnes (règle de Statistique Canada relative à la protection de la vie privée). 
TABLEAU 3

Sommaire des taux de dépistage par RSOS et endoscopie dans les régions sanitaires selon la province

\begin{tabular}{|c|c|c|c|c|c|}
\hline \multirow[t]{2}{*}{ Province } & \multirow{2}{*}{$\begin{array}{c}\text { Régions sanitaires } \\
\text { n (\%) }\end{array}$} & \multicolumn{2}{|c|}{ Fourchette du RSOS ${ }^{b}$} & \multicolumn{2}{|c|}{$\begin{array}{l}\text { Fourchette de } \\
\text { l'endoscopie }^{\text {b }}\end{array}$} \\
\hline & & $\begin{array}{c}\text { Année } \\
\text { dernière }\end{array}$ & Jamais & $\begin{array}{l}5 \text { dernières } \\
\text { années }\end{array}$ & Jamais \\
\hline Globala $^{a}$ & 43 & 2,4 à 21,5 & 54,3 à 89,2 & 4,2 à 16,5 & 81,1 à 94,3 \\
\hline $\begin{array}{l}\text { Terre-Neuve } \\
\text { et Labrador }\end{array}$ & $6(100 \%)$ & 2,8 à 5,4 & 83,6 à 89,1 & 4,8 à 12,0 & 84,6 à 94,3 \\
\hline Ontario & $14(37,8 \%)$ & 3,9 à 13,1 & 62,5 à 85,3 & 8,2 à 16,5 & 81,1 à 88,4 \\
\hline Saskachewan & $7(63,6 \%)$ & 3,8 à 21,5 & 54,3 à 85,5 & 7,5 à 10,8 & 82,6 à 90,8 \\
\hline $\begin{array}{l}\text { Colombie- } \\
\text { Britannique }\end{array}$ & $16(100 \%)$ & 2,4 à 15,1 & 56,5 à 89,2 & 4,2 à 13,4 & 83,4 à 93,8 \\
\hline
\end{tabular}

a Toutes les régions sanitaires/provinces combinées.

${ }^{\mathrm{b}}$ Les valeurs des fourchettes sont des pourcentages.

différence n'est ressortie entre les régions rurales et urbaines. Les taux de dépistage par RSOS provinciaux sont plus élevés pour les résidents de la Colombie-Britannique comparativement à ceux de Terre-Neuve. Le taux de dépistage par RSOS dans les régions sanitaires varie dans chaque province (tableau 3). Dans l'ensemble des 43 régions sanitaires de toutes les provinces, les taux de dépistage par RSOS au cours de l'année précédente varient de $2,4 \%$ à $21,5 \%$ et les taux des personnes sondées n'ayant jamais subi de dépistage par RSOS variaient de $54,3 \%$ à $89,2 \%$. Par comparaison, les taux globaux d'utilisation du RSOS s'établissent comme suit : 9,1 \% pour l'année précédente, $6 \%$ entre un et deux ans auparavant, $11,8 \%$ pour trois ans et plus; $69,3 \%$ n'ont jamais subi de RSOS (données non fournies).

\section{Taux de dépistage par endoscopie}

Le tableau 4 présente les taux de dépistage par endoscopie selon l'intervalle de temps et des caractéristiques sociodémographiques, cliniques et géographiques. Les taux de dépistage par endoscopie globaux sont de $8,8 \%$ dans les cinq dernières années et de $1,5 \%$ entre six à dix ans; 88,7\% des personnes sondées n'ont jamais subi de dépistage par endoscopie. Les taux de dépistage par endoscopie au cours des cinq dernières années sont plus élevés chez les enquêtés ayant une maladie entérite et les enquêtés âgés de 65 ans et plus; les taux sont les plus faibles dans les groupes d'âge des 50 à 59 ans et des 90 à 100 ans. Nous n'avons constaté aucune différence entre les régions rurales et urbaines. Les taux de dépistage par endoscopie provinciaux sont plus élevés pour les résidents de TerreNeuve par rapport à ceux de la ColombieBritannique. Les taux de dépistage par endoscopie dans les régions sanitaires varient au sein de chaque province (tableau 3). Dans l'ensemble des 43 régions sanitaires (c.-à-d. toutes les provinces), les taux de dépistage par endoscopie au cours des cinq dernières années varient de $4,2 \%$ à $16,5 \%$ et les taux des personnes sondées n'ayant jamais subi de dépistage par endoscopie varient de $81,1 \%$ à $94,3 \%$. Par comparaison, les taux globaux d'utilisation de l'endoscopie sont de $16,7 \%$ dans les cinq années précédentes et de 3,9\% entre jamais subi d'endoscopie (données non fournies).

Le tableau 5 montre que $15,1 \%$ des personnes sondées observent les lignes directrices relatives au dépistage par RSOS. La figure 2 montre que les taux d'observation de ces lignes directrices sont plus élevés dans les régions sanitaires du Sud de la Colombie-Britannique et dans quelques régions sanitaires de la Saskatchewan et de l'Ontario. Les proportions de personnes sondées n'ayant jamais subi de RSOS dans l'ensemble des régions sanitaires (figure 3) six à dix ans; 75,6\% des interrogés n'ont

\section{Observation des lignes directrices relatives au dépistage par RSOS}

sont plus élevées dans les régions sanitaires de l'Est de la Colombie-Britannique, dans la région de Saskatoon, au Nordest de l'Ontario et dans des parties de Terre-Neuve.

\section{Observation des lignes directrices relatives au dépistage par endoscopie}

En tout, 20,6 \% des personnes sondées observaient les lignes directrices relatives au dépistage par endoscopie (tableau 5). La figure 4 montre que les taux régionaux d'observation de ces lignes directrices sont plus élevés dans le Sud-est de la Colombie-Britannique, le Sud de l'Alberta, le Nord de l'Ontario et dans des parties de Terre-Neuve. Les proportions de personnes sondées n'ayant jamais subi d'endoscopie (figure 5) étaient plus élevées en Colombie-Britannique et dans les parties de Terre-Neuve. Aucune tendance géographique claire n'est ressortie pour la Saskatchewan puisque moins de la moitié des régions sanitaires de la province ont été échantillonnées.

\section{Observation des lignes directrices en vigueur relatives au dépistagé du CCR}

Dans la population de la présente étude, $30,1 \%$ des personnes sondées observent les lignes directrices en vigueur relatives au dépistage du CCR et 69,9\% des personnes sondées ne les observent pas (tableau 5). La figure 6 montre que les taux d'inobservation sont plus élevés dans la plupart des régions sanitaires de la ColombieBritannique et plus faibles dans de nombreuses régions sanitaires de l'Ontario. Les taux d'inobservation pour Terre-Neuve et la Saskatchewan varient selon la région sanitaire.

\section{Discussion}

L'objectif principal de la présente étude fondée sur une population canadienne est d'améliorer notre connaissance de la mesure dans laquelle les Canadiens de 50 ans et plus subissent un dépistage du CCR. Nos résultats montrent que les taux de dépistage du CCR par RSOS et endoscopie sont faibles et susceptibles de varier beaucoup selon la région géographique. Les taux de dépistage provinciaux (à Terre-Neuve et en ColombieBritannique) révèlent que jusqu'à 85,4\% et $91,4 \%$ des résidents n'ont jamais subi 
TABLEAU 4

Taux de dépistage par endoscopie selon l'intervalle de temps en fonction des caractéristiques sociodémographiques, cliniques et géographiques $\left(n^{\mathrm{a}}=13949\right)$

\begin{tabular}{|c|c|c|c|c|c|c|c|c|c|c|c|c|}
\hline \multirow{4}{*}{$\begin{array}{l}\text { Caractéristique } \\
\text { GLOBAL }\end{array}$} & \multicolumn{12}{|c|}{ Dernier dépistage par endoscopie déclaréb } \\
\hline & \multicolumn{3}{|c|}{0 à 5 ans } & \multicolumn{3}{|c|}{6 à 10 ans } & \multicolumn{3}{|c|}{10 ans et plus } & \multirow{2}{*}{\multicolumn{3}{|c|}{$\begin{array}{l}\text { Jamais } \\
\text { Taux } \\
\text { IC } 95 \%\end{array}$}} \\
\hline & \multirow{2}{*}{$\begin{array}{l}\text { Taux } \\
\mathbf{8 , 8}\end{array}$} & \multicolumn{2}{|c|}{ IC $95 \%$} & \multirow{2}{*}{$\begin{array}{l}\text { Taux } \\
1,5\end{array}$} & \multicolumn{2}{|c|}{ IC $95 \%$} & \multirow{2}{*}{$\begin{array}{l}\text { Taux } \\
1,0\end{array}$} & \multicolumn{2}{|c|}{ IC $95 \%$} & & & \\
\hline & & 8,2 & 9,5 & & 1,2 & 1,8 & & 0,8 & 1,2 & 88,7 & 88,0 & 89,4 \\
\hline \multicolumn{13}{|l|}{ Sexe } \\
\hline Masculin & 9,0 & 8,0 & 10,0 & 1,3 & 0,9 & 1,7 & 1,1 & 0,7 & 1,5 & 88,7 & 87,6 & 89,7 \\
\hline Féminin & 8,7 & 7,8 & 9,6 & 1,7 & 1,3 & 2,1 & 0,9 & 0,7 & 1,2 & 88,7 & 87,7 & 89,7 \\
\hline \multicolumn{13}{|l|}{ Âge (ans) } \\
\hline 50 à 64 & 8,1 & 7,3 & 9,0 & 1,1 & 0,8 & 1,5 & 0,6 & 0,4 & 0,9 & 90,1 & 89,2 & 91,0 \\
\hline 65 et plus & 10,0 & 8,9 & 11,0 & 2,0 & 1,6 & 2,5 & 1,6 & 1,2 & 2,0 & 86,5 & 85,3 & 87,6 \\
\hline 50 à 59 & 7,3 & 6,4 & 8,3 & 1,0 & 0,7 & 1,4 & 0,5 & 0,3 & 0,8 & 91,1 & 90,1 & 92,2 \\
\hline 60 à 69 & 10,4 & 9,2 & 11,6 & 1,8 & 1,2 & 2,4 & 1,0 & 0,6 & 1,4 & 86,9 & 85,6 & 88,2 \\
\hline 70 à 79 & 10,3 & 8,8 & 11,8 & 2,1 & 1,6 & 2,7 & 1,7 & 0,9 & 2,4 & 86,0 & 84,3 & 87,7 \\
\hline 80 à 89 & 9,7 & 6,6 & 12,8 & 1,6 & 0,7 & 2,5 & 2,1 & 1,3 & 3,0 & 86,6 & 83,4 & 89,8 \\
\hline 90 à 100 & 2,7 & $\mathrm{n} / \mathrm{a}$ & 5,7 & $\mathrm{n} / \mathrm{a}$ & n/a & $\mathrm{n} / \mathrm{a}$ & $\mathrm{n} / \mathrm{a}$ & $\mathrm{n} / \mathrm{a}$ & $\mathrm{n} / \mathrm{a}$ & 94,6 & 90,5 & 98,8 \\
\hline \multicolumn{13}{|l|}{ Régoion de résidence } \\
\hline Urbaine & 9,0 & 8,2 & 9,8 & 1,5 & 1,2 & 1,8 & 0,9 & 0,7 & 1,1 & 88,6 & 87,7 & 89,4 \\
\hline Rurale & 8,2 & 7,1 & 9,4 & 1,4 & 0,9 & 1,9 & 1,2 & 0,6 & 1,9 & 89,1 & 87,8 & 90,4 \\
\hline \multicolumn{13}{|l|}{ Études } \\
\hline$<$ Niveau secondaire & 8,2 & 7,1 & 9,2 & 1,6 & 1,1 & 2,0 & 1,0 & 0,6 & 1,4 & 89,3 & 88,1 & 90,6 \\
\hline \multicolumn{13}{|l|}{ Diplôme d'études } \\
\hline secondaires & 8,4 & 6,8 & 10,0 & 1,3 & 0,7 & 1,9 & 1,0 & 0,5 & 1,4 & 89,3 & 87,6 & 91,1 \\
\hline Études postsecondaires & 10,8 & 7,6 & 13,9 & 2,6 & 0,5 & 4,7 & 1,7 & $\mathrm{n} / \mathrm{a}$ & 3,7 & 84,9 & 81,3 & 88,6 \\
\hline \multicolumn{13}{|l|}{ Diplôme d'études } \\
\hline \multicolumn{13}{|l|}{ Revenu du ménagé } \\
\hline Faible à moyen inférieur & 8,8 & 7,5 & 10,2 & 1,6 & 1,1 & 2,0 & 1,2 & 0,8 & 1,7 & 88,4 & 87,0 & 89,8 \\
\hline Moyen supérieur & 8,5 & 7,4 & 9,6 & 1,7 & 1,2 & 2,1 & 1,1 & 0,6 & 1,6 & 88,7 & 87,5 & 90,0 \\
\hline Élevé & 9,1 & 7,7 & 10,4 & 1,5 & 0,8 & 2,2 & 0,7 & 0,4 & 1,0 & 88,7 & 87,2 & 90,2 \\
\hline \multicolumn{13}{|l|}{ Maladie entérique } \\
\hline Oui & 23,2 & 16,5 & 29,9 & 4,1 & 1,6 & 6,6 & $\mathrm{n} / \mathrm{a}$ & $\mathrm{n} / \mathrm{a}$ & $\mathrm{n} / \mathrm{a}$ & 71,0 & 63,7 & 78,2 \\
\hline Non & 8,6 & 7,9 & 9,2 & 1,4 & 1,2 & 1,7 & 1,0 & 0,8 & 1,2 & 89,0 & 88,3 & 89,7 \\
\hline \multicolumn{13}{|l|}{ Province de résidence ${ }^{c}$} \\
\hline $\begin{array}{l}\text { Terre-Neuve } \\
\text { et Labrador }\end{array}$ & 9,0 & 7,3 & 10,8 & 1,7 & 0,7 & 2,7 & 1,2 & 0,5 & 2,0 & 88,1 & 86,1 & 90,0 \\
\hline Colombie-Britannique & 6,5 & 5,7 & 7,4 & 1,2 & 0,8 & 1,6 & 0,8 & 0,5 & 1,2 & 91,4 & 90,5 & 92,4 \\
\hline
\end{tabular}

Les pourcentages des taux représentent des données pondérées.

${ }^{a}$ Nombre de personnes sondées ayant fourni des renseignements sur le dépistage par endoscopie et représentant 2029620 Canadiens.

Les taux sont fondés sur les réponses valides (excluant les valeurs manquantes).

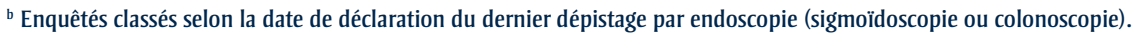

Les endoscopies exécutées à d'autres fins que le dépistage sont exclues.

c Les taux provinciaux pour l'Ontario et la Saskatchewan ne sont pas indiqués parce que nous ne disposons pas de données pour toutes les régions sanitaires.

n.d.= non disponible parce que les cellules de données non pondérées contenaient moins de 5 personnes (règle de Statistique Canada relative à la protection de la vie privée). 
TABLEAU 5

Fréquence des trois variables de résultats touchant l'observation

\begin{tabular}{|c|c|c|}
\hline Résultat & $\mathrm{n}$ & $\%^{a}$ \\
\hline \multicolumn{3}{|c|}{ Observation des lignes directrices relatives au dépistage par RSOS $^{b}$} \\
\hline Oui & 356535 & 15,1 \\
\hline Non & 2005605 & 84,9 \\
\hline \multicolumn{3}{|c|}{ Observation des lignes directrices relatives au dépistage par endoscopie ${ }^{c}$} \\
\hline Oui & 490128 & 20,6 \\
\hline Non & 1890047 & 79,4 \\
\hline \multicolumn{3}{|c|}{ Observation des lignes directrices en vigueur relatives au dépistage du $\mathbf{C C R}^{\mathbf{d}}$} \\
\hline Oui & 720899 & 30,1 \\
\hline Non $^{e}$ & 1673225 & 69,9 \\
\hline
\end{tabular}

Tous les chiffres sont pondérés.

Comprend les procédures pour toutes les indications.

a Fondé sur les réponses valides (exclut les valeurs manquantes).

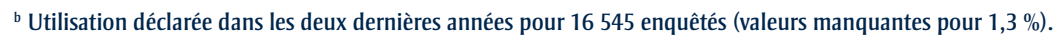

c Utilisation déclarée dans les dix dernières années pour 16648 enquêtés (valeurs manquantes pour 0,6 \%).

d Utilisation déclarée de la RSOS dans les deux dernières années ou de l'endoscopie dans les dix dernières années pour 16747 enquêtés.

${ }^{\text {e }}$ Comprend 1,3\% de personnes sondées classées selon une seule procédure.

FIGURE 2

Taux d'observation des lignes directrices relatives au dépistage par RSOS dans les régions

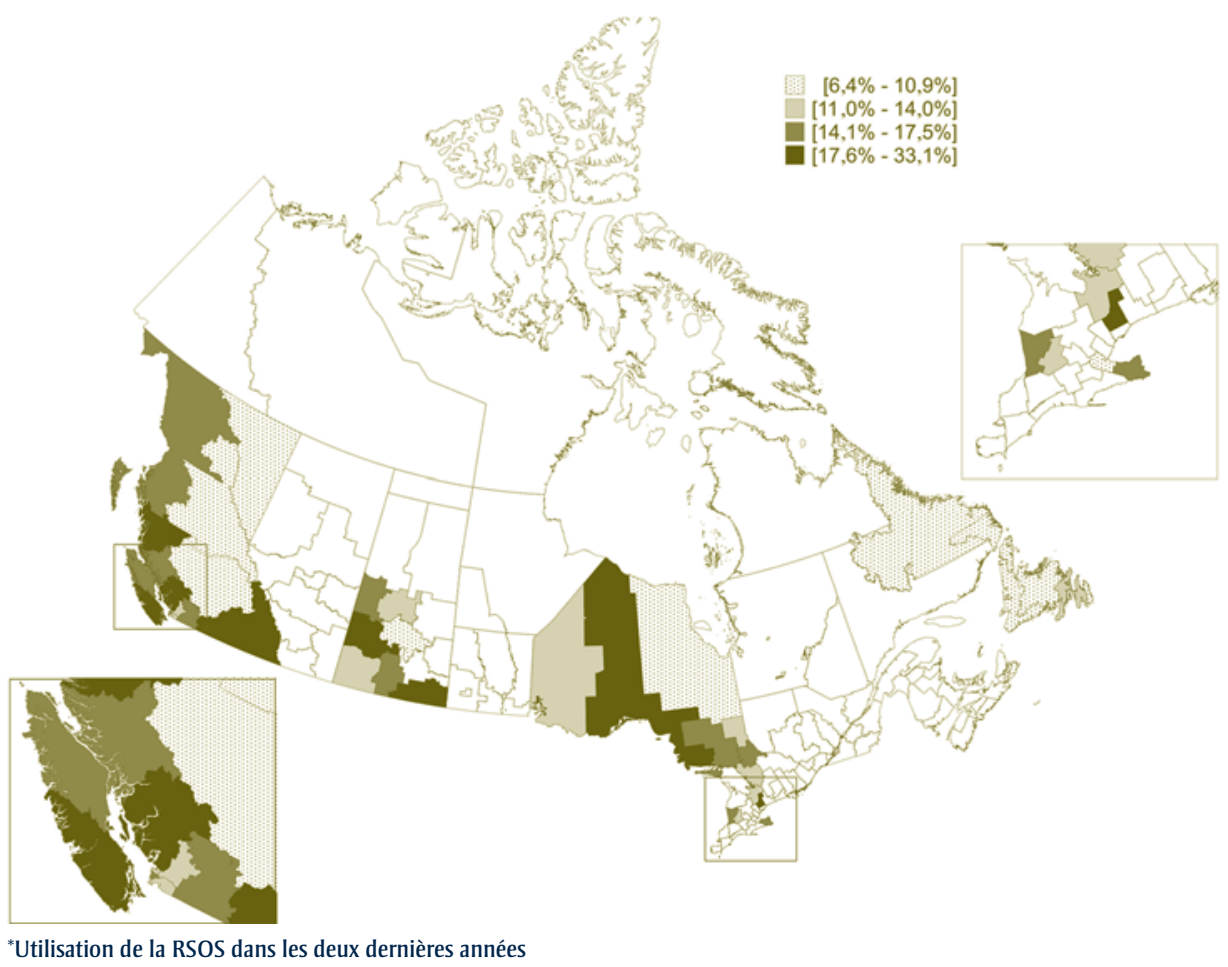


FIGURE 3

Taux des personnes sondées n'ayant jamais utilisé la RSOS dans les régions sanitaires

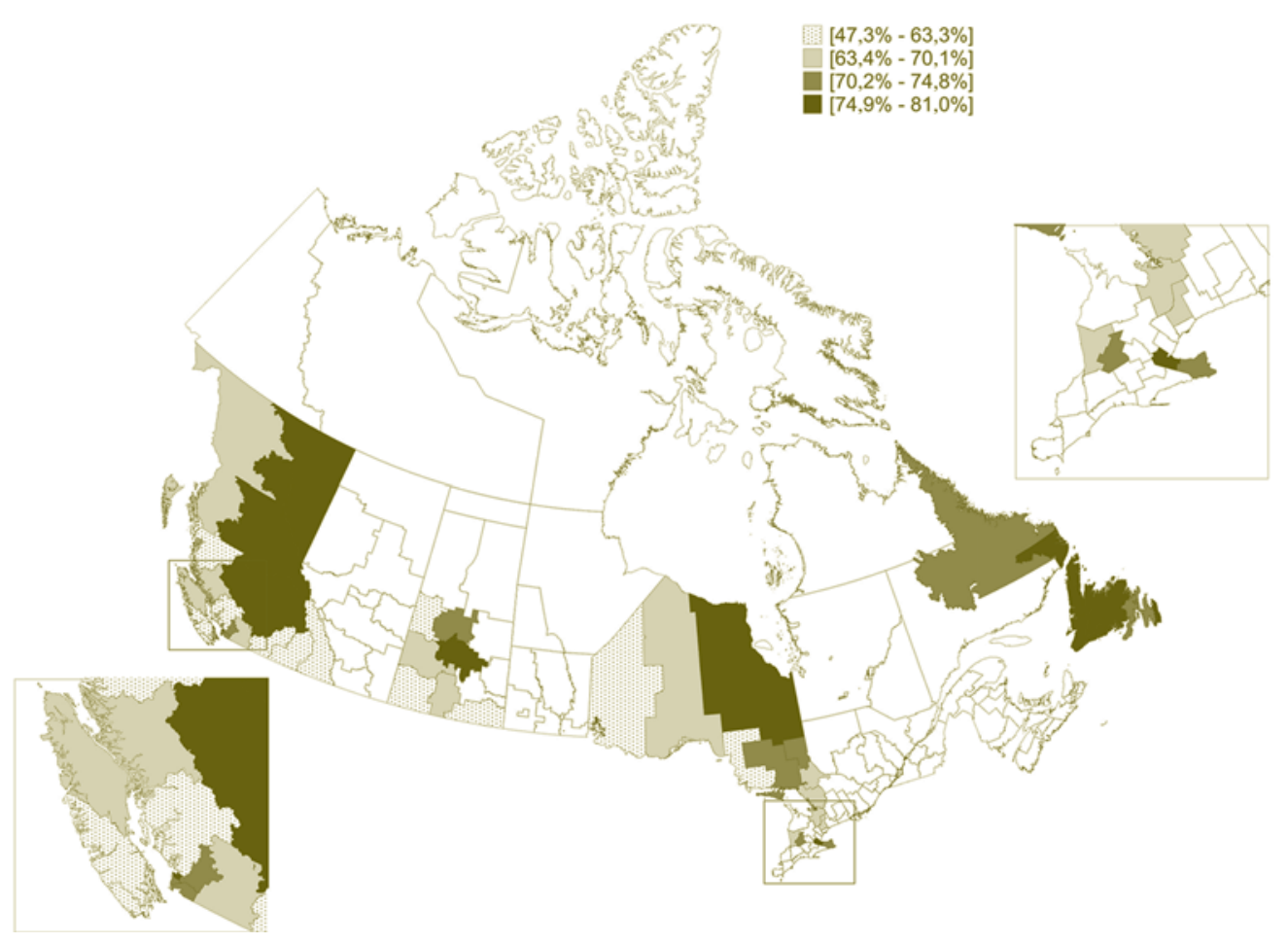

FIGURE 4

Taux d'observation des lignes directrices relatives au dépistage par endoscopie dans les régions sanitaires*

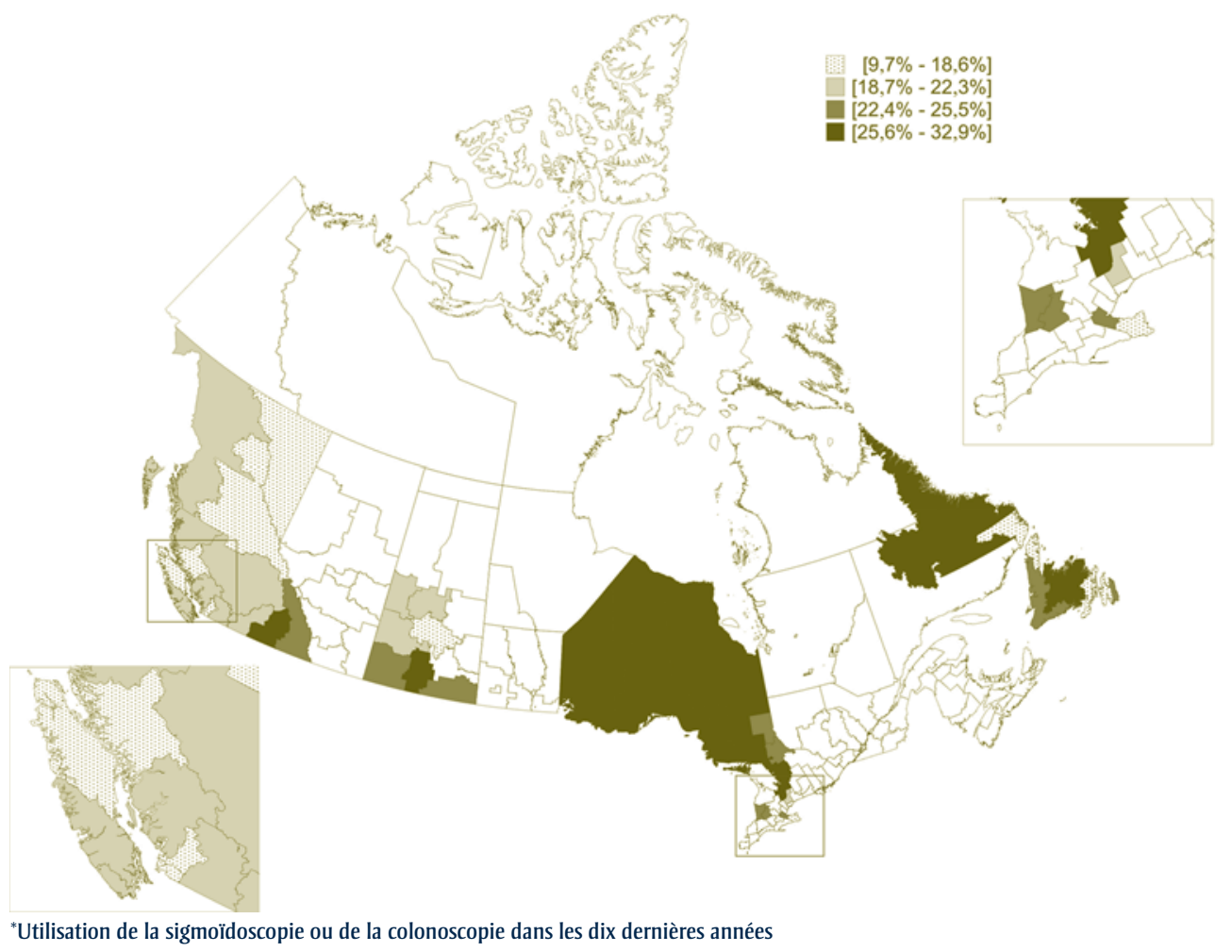


FIGURE 5

Taux des personnes sondées n'ayant jamais utilisé l'endoscopie dans les régions sanitaires

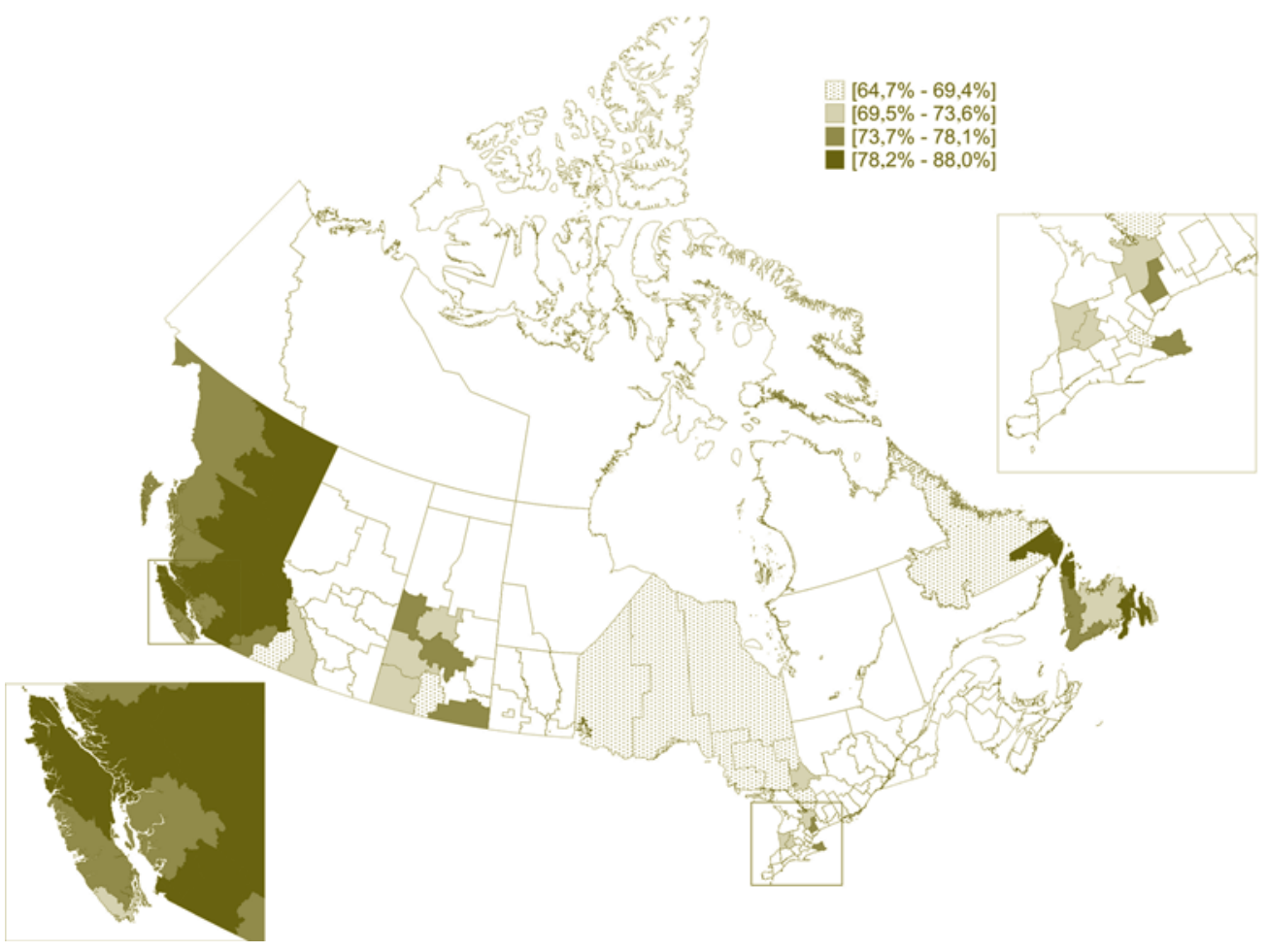

FIGURE 6

Taux d'inobservation des lignes directrices en vigueur relatives au dépistage du cancer colorectal dans les régions sanitaires*

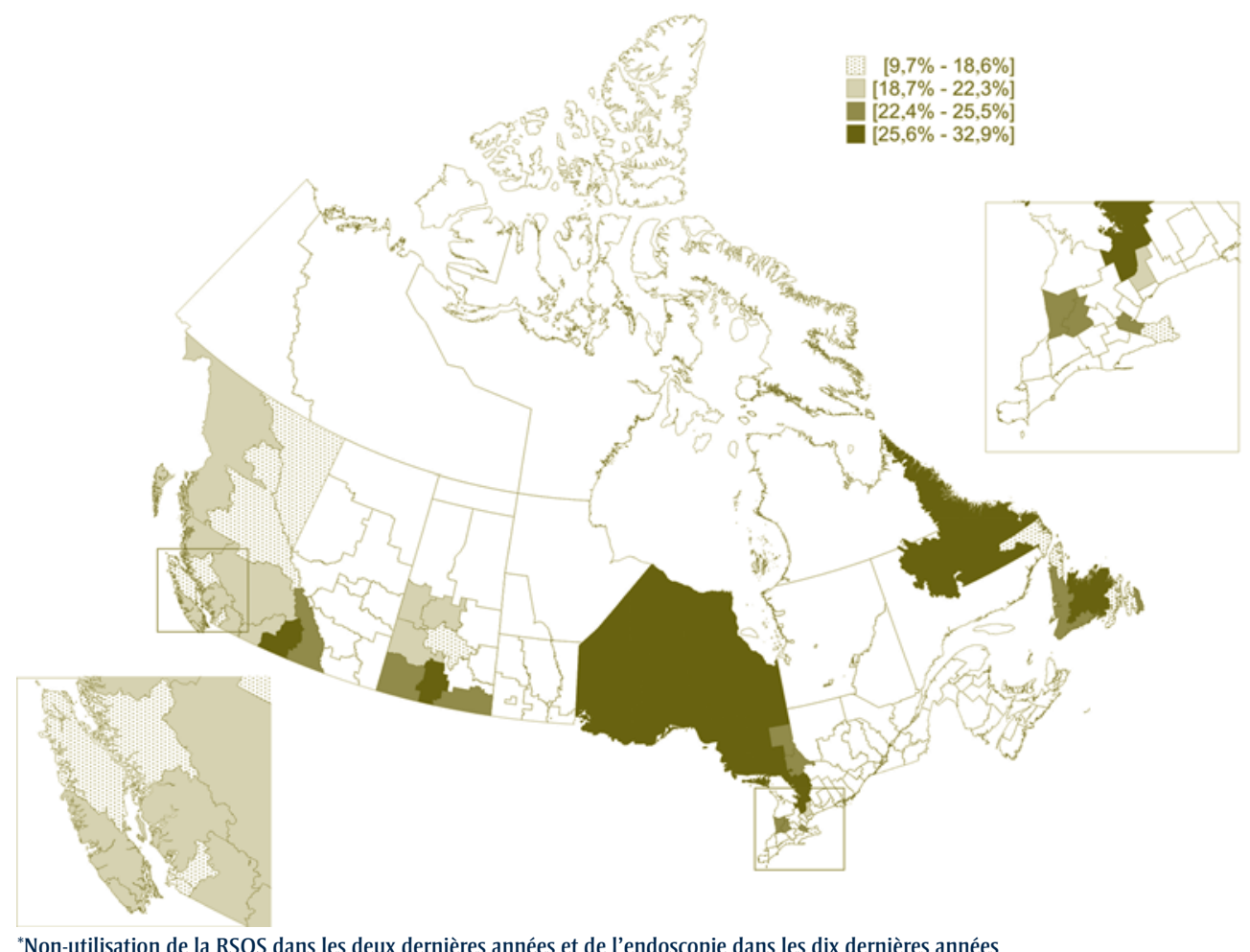

${ }^{*}$ Non-utilisation de la RSOS dans les deux dernières années et de l'endoscopie dans les dix dernières années 
de dépistage par RSOS et endoscopie, respectivement. Les taux de dépistage dans les régions sanitaires (de l'Ontario et de la Saskatchewan) sont faibles eux aussi, $85,5 \%$ et $90,8 \%$ de la population n'ayant jamais subi de dépistage par RSOS et endoscopie, respectivement. Les variations géographiques importantes rappellent les constatations d'une étude basée en Alberta ${ }^{21}$ et de plusieurs études menées aux ÉtatsUnis qui montrent d'importantes différences entre régions et États à l'égard de l'utilisation des tests de dépistage du $\mathrm{CCR}^{22,23}$. Les variations géographiques liées à l'utilisation des services de prévention pour la santé sont évidentes pour d'autres formes de dépistage au Canada. Les taux d'utilisation du test Papanicolaou (Pap) dans les régions sanitaires de l'Ontario varieraient de $12 \%$ à $74 \%{ }^{24}$ tandis que dans l'ensemble du Canada, les taux provinciaux varient entre $70 \%$ et $88 \%^{25}$. De même, des données d'enquêtes montrent que les taux provinciaux de dépistage du cancer du sein au Canada varient d'environ $9 \%$ au Nunavut à $67 \%$ au Nouveau-Brunswick ${ }^{25}$.

Les taux de dépistage du CCR varient aussi en fonction de l'âge. Les enquêtés de 65 ans et plus subissent plus souvent des tests de dépistage par RSOS et endoscopie que leurs cadets, comme d'autres auteurs l'ont constaté $^{26-34}$. Les enquêtés de 50 à 59 ans sont moins susceptibles de déclarer avoir subi un dépistage du CCR, ce qui semble révéler une acceptation à retardement des recommandations relatives au dépistage du CCR soit chez les médecins, soit chez leurs patients plus jeunes, ou les deux, puisqu'au moment de l'enquête, aucun programme de dépistage du CCR n'était en vigueur. En outre, les taux de dépistage par RSOS et endoscopie fléchissent dans le groupe des 80 à 89 ans et, pour l'endoscopie, ils chutent de façon spectaculaire dans le groupe des 90 à 100 ans. Ces constatations sont peutêtre un reflet du modèle de rentabilité proposé pour la population canadienne qui recommande de cesser le dépistage à l'âge de 74 ans $^{3,35}$.

Nous n'avons relevé aucune différence significative des taux de dépistage du CCR selon la région de résidence urbaine ou rurale, ce qui donne à penser que la variation des taux de dépistage n'est pas due à la disponibilité de ressources sanitaires. Nous aurions pu croire que les régions rurales auraient été défavorisées quant à l'accessibilité de la sigmoïdoscopie et de la colonoscopie et, par conséquent, plus susceptibles d'employer la RSOS, plus facilement disponible. Notre constatation corrobore celle rapportée dans une autre étude canadienne qui a relevé des taux d'utilisation de procédures de dépistage du CCR semblables dans les régions rurales et urbaines $^{21}$. Une explication possible de l'absence de variabilité entre les régions résidentielles peut tenir aux similitudes régionales en fait de style d'exercice de la médecine. Puisque la RSOS et l'endoscopie sont considérées comme les principales modalités de dépistage, les médecins qui adhèrent au dépistage par endoscopie peuvent recommander l'endoscopie peu importe que les installations soient situées hors de la région de résidence du patient.

Les taux d'utilisation de la RSOS sont légèrement plus élevés pour la période entre un et deux ans auparavant et l'an dernier tandis que les taux d'utilisation de l'endoscopie sont multipliés presque par six pour la dernière décennie. Ces tendances indiquent non seulement une augmentation régulière du dépistage du CCR, elles peuvent illustrer aussi une transition de la RSOS en faveur de l'endoscopie comme modalité principale de dépistage du $\mathrm{CCR}^{21,34,36-39}$. Ces constatations devraient alerter les décideurs et les responsables de l'élaboration des politiques d'une demande accrue imminente pour le dépistage par endoscopie puisqu'à l'heure actuelle, les ressources sont insuffisantes pour satisfaire à cette demande ${ }^{35}$.

Au Canada en 2003, 78,4 \% et 88,7 \% des personnes sondées n’avaient jamais subi de dépistage par RSOS ou endoscopie, respectivement. Ces constatations rappellent celles d'études basées sur des populations aux États-Unis qui ont révélé que jusqu'à $65 \%$ des personnes sondées n'avaient jamais subi l'un ou l'autre de ces examens ${ }^{7,11,36}$ et d'une étude canadienne qui rapporte que jusqu'à $77 \%$ des personnes sondées n’ont jamais subi de dépistage du $\mathrm{CCR}^{14}$. De même, dans le cadre d'une étude de suivi, après six ans, portant sur les bénéficiaires de l'Ontario, près de $80 \%$ des personnes admissibles au dépistage du CCR, âgées de 50 ans à 59 ans, n'avaient pas subi d'examen du gros intestin $^{12}$. Nos constatations révèlent non seulement la sous-utilisation flagrante du dépistage du CCR, elles montrent également que les taux de dépistage du CCR sont nettement plus faibles que ceux du dépistage du cancer du sein ou du $\operatorname{col}^{8,37,40}$. En 2003, la proportion des femmes admissibles qui ont subi un test de dépistage conformément aux recommandations des lignes directrices s'établissait à $79 \%$ pour le test Pap et $61 \%$ pour la mammographie, ce dernier taux représentant une augmentation par rapport à $53 \%$ en $2001^{25}$. L’augmentation des taux de dépistage du cancer du sein peut s'expliquer par le nombre grandissant de femmes inscrites à des programmes de dépistage du cancer du sein organisés par les provinces, programmes que toutes les provinces canadiennes ont adoptés ${ }^{41}$. Les initiatives multimodales qui se sont révélées efficaces pour améliorer les taux de dépistage du cancer du sein pourraient être efficaces pour augmenter les taux de dépistage du CCR. Indubitablement, la prestation du dépistage du CCR dans le contexte d'un programme organisé peut se révéler avantageuse en favorisant la sensibilisation au dépistage du CCR, en particulier chez les plus jeunes, et le suivi du dépistage du CCR selon les recommandations des lignes directrices pour les Canadiens présentant un risque moyen.

Il est important de prendre en compte plusieurs limitations et plusieurs forces en interprétant nos constatations. Les taux de dépistage par endoscopie peuvent avoir été surestimés parce que des personnes interrogées souffrant de maladies entériques peuvent avoir déclaré une procédure de dépistage. Étant donné que la maladie intestinale inflammatoire constitue un facteur de risque au développement du CCR, on conseille aux individus atteints par cette dernière de subir une procédure de surveillance par colonoscopie à intervalles réguliers comparativement à ceux qui sont moyennement à risque. Toutefois, cela n'aurait pas modifié de façon appréciable les taux de dépistage parce que 
moins de $1 \%$ de la population de l'étude aurait été mal classifiée (3,6\% des interrogés souffraient de maladies entériques, dont $23 \%$ avaient subi un dépistage par endoscopie au cours des cinq dernières années). L'observation des lignes directrices relatives au dépistage par endoscopie peut avoir été surestimée si les personnes qui ont subi une sigmoïdoscopie au-delà des cinq années recommandées ont été incluses. L'observation des lignes directrices en vigueur relatives au dépistage du CCR peut avoir été sous-estimée puisque 1,3\% des interrogés ont été classés dans la catégorie d'inobservation en fonction d'une seule procédure. Les taux de dépistage du CCR peuvent avoir été légèrement sousestimés puisque certains des interrogés âgés de 50 ans qui ont déclaré n'avoir pas subi de dépistage au moment de l'enquête peuvent avoir subi un test de dépistage avant la fin de leur $50^{\mathrm{e}}$ année. Compte tenu de la grande variabilité géographique, nous ne pouvons probablement pas généraliser les résultats aux provinces canadiennes pour lesquelles des données sur le dépistage du CCR ne sont pas disponibles.

Il est difficile du point de vue méthodologique de déterminer la mesure dans laquelle le dépistage du CCR est exécuté à l'échelle nationale. Si les études fondées sur des bases de données administratives ne peuvent établir de distinction entre des examens de dépistage et ceux qui sont exécutés pour d'autres indications, des enquêtes comme le cycle 2.1 de l'ESCC renferment des réponses qui permettent de déterminer l'indication de la procédure. Par contraste, les enquêtes qui reposent sur l'autodéclaration peuvent difficilement faire la distinction entre la sigmoïdoscopie et la colonoscopie. Cependant, il est possible d'obtenir une bonne sensibilité et une bonne spécificité pour l'utilisation autodéclarée des procédures lorsque les deux procédures sont regroupées ${ }^{42,43}$, comme cela a été fait dans l'ESCC.

Les forces de l'étude comprennent 1) la définition de l'observation des lignes directrices en vigueur relatives au dépistage du CCR en fonction de critères multiples pour obtenir un instantané (aperçu) de la proportion de la population qui a subi un dépistage du CCR; et 2) la détermination des résultats dans une population où plus de $96 \%$ des gens courent le risque de développer un CCR. Enfin, les taux selon la région géographique sont susceptibles de rester stables sur des intervalles de temps courts compte tenu que $4,1 \%$ seulement des Canadiens âgés de 45 ans et plus changent de province de résidence dans une année donnée $e^{44}$.

\section{Conclusion}

En résumé, la plupart des interrogés présentant un risque moyen n'avaient jamais subi de dépistage du CCR, soit par RSOS soit par endoscopie, puisque près de $70 \%$ des interrogés n'observaient pas les lignes directrices en vigueur relatives au dépistage. Les taux plus élevés d'inobservation des lignes directrices relatives au dépistage du CCR chez les enquêtés âgés de 50 à 59 ans semblent indiquer l'adhésion à retardement des recommandations en question chez les médecins et les Canadiens plus jeunes présentant un risque moyen. Nous ne pouvons pas déterminer pourquoi les taux de dépistage sont plus faibles dans la plupart des régions de la Colombie-Britannique. L'utilisation plus grande de l'endoscopie par opposition à la RSOS porte à croire qu'elle peut être employée de plus en plus en guise de principale stratégie de dépistage du CCR. L'absence de différence entre régions urbaines et rurales semble indiquer que l'absence de ressources dans les régions rurales n'est pas un obstacle au dépistage du CCR. Les taux de dépistage très faibles révélés dans la présente étude, de pair avec la preuve que le dépistage du CCR peut réduire l'incidence et la mortalité du CCR donnent à penser que des initiatives multimodales s'imposent pour sensibiliser davantage les Canadiens au dépistage du CCR et pour augmenter les taux d'utilisation des modalités de dépistage.

\section{Remerciements}

La présente recherche a bénéficié d'une subvention des Instituts de recherche en santé du Canada (IRSC), MOP 77666.
Maida J. Sewitch, Ph.D., bénéficie d'un soutien comme chercheure de la Société canadienne du cancer grâce à une subvention de l'Institut national du cancer du Canada.

Les auteurs ont obtenu l'approbation du conseil de l'éthique en recherche de l'Institut de recherche du Centre universitaire de santé McGill et la permission de Statistique Canada avant le début de l'étude.

\section{References}

1. Société canadienne du cancer/Institut national du cancer du Canada. Statistiques canadiennes sur le cancer 2008, Toronto, 2008.

2. Agence de la santé publique du Canada. Réduire la mortalité associée au cancer colorectal au Canada grâce au dépistage, 2002. URL : http://www.phac-aspc.gc.ca/ publicat/ncccs-cndcc/ccsrec-fra.php .

3. Leddin D. The Canadian Association of Gastroenterology position on colon cancer screening. Can J Gastroenterol. 2003;17(2): 133-134.

4. Société canadienne du cancer. Dépistage du cancer colorectal, 2004. URL : http://www. cancer.ca/ccs/internet/standard/0,,3649_ 10175_74577648_langId-fr,00.html .

5. U.S. Preventive Services Task Force. Screening for colorectal cancer: recommendations and rationale. Ann Intern Med. 2002; 137(2):129-131.

6. Winawer $\mathrm{S}$, Fletcher $\mathrm{R}$, Rex $\mathrm{D}$ et al. Colorectal cancer screening and surveillance: clinical guidelines and rationaleupdate based on new evidence. Gastroenterology. 2003;124:544-560.

7. Ata A, Elzey JD, Insaf TZ, Grau AM, Stain SC, Ahmed NU. Colorectal cancer prevention: adherence patterns and correlates of tests done for screening purposes within United States populations. Cancer Detect Prev. 2006;30(2):134-143.

8. Increased use of colorectal cancer tests United States, 2002 and 2004. MMWR Morb Mortal Wkly Rep. 2006;55(11):308-311. 
9. McGlynn EA, Asch SM, Adams J, et al. The quality of health care delivered to adults in the United States. N Engl J Med. 2003; 348(26):2635-2645.

10. Etzioni DA, Ponce NA, Babey SH et al. A population-based study of colorectal cancer test use: results from the 2001 California Health Interview Survey. Cancer. 2004; $101(11): 2523-2532$.

11. McQueen A, Vernon SA, Meissner HI, Klabunde $\mathrm{CN}$. Are there gender differences in colorectal cancer test use prevalence and correlates? Cancer Epidemiology, Biomarkers \& Prevention. 2006;15(4): 782-791.

12. Rabeneck L, Paszat LF. A population-based estimate of the extent of colorectal cancer screening in Ontario. Am J Gastroenterol. 2004;99(6):1141-1144.

13. Vinden C, Schultz S, Rabeneck L. ICES Research Atlas: Use of Large Bowel Procedures in Ontario, Toronto, Institute for Clinical Evaluative Sciences (ICES), 2004.

14. Ramji F, Cotterchio M, Manno M, Rabeneck L, Gallinger S. Association between subject factors and colorectal cancer screening participation in Ontario, Canada. Cancer Detection and Prevention. 2005;29(3): 221-226.

15. McGregor SE, Hilsden RJ, Li FX, Bryant HE, Murray A. Low uptake of colorectal cancer screening 3 years after release of national recommendations for screening. Am J Gastroenterol. 2007;102(8):1727-1735.

16. Statistique Canada. Le cycle 2.1 de l'Enquête sur la santé dans les collectivités canadiennes, 2003. URL : http:// www.statcan.ca/francais/concepts/health/ cycle2_1/cchsinfo_f.htm.

17. Statistique Canada. Le cycle 2.1 de l'Enquête sur la santé dans les collectivités canadiennes, 2002.

18. Statistique Canada. Régions sociosanitaires : limites et correspondance avec la géographie du recensement, 2003, $\mathrm{n}^{\circ} 82$ 402-XIE au catalogue.

19. Statistique Canada. Enquête sur la santé dans les collectivités canadiennes 2003 : Fichier de microdonnées à grande diffusion - Guide de l'utilisateur, 2005:22-32.

20. SAS Version 8.02. Cary, North Carolina, USA, 2001.

21. Hilsden RJ. Patterns of use of flexible sigmoidoscopy, colonoscopy and gastroscopy: a population-based study in a Canadian province. Can J Gastroenterol. 2004;18(4):213-2119.

22. Ko CW, Kreuter W, Baldwin L-M. Persistent demographic differences incolorectal cancer screening utilization despite Medicare reimbursement. BMC Gastroenterol. 2005;5(1):10.

23. Cooper GS, Koroukian SM. Geographic variation among Medicare beneficiaries in the use of colorectal carcinoma screening procedures. Am J Gastroenterol. 2004; 99(8):1544-1550.

24. Fehringer G, Howlett R, Cotterchio M, Klar N, Majpruz-Moat V, Mai V. Comparison of papanicolaou (Pap) test rates across Ontario and factors associated with cervical screening. Can J Public Health. 2005;96(2):140-144.

25. Société canadienne du cancer/Institut national du cancer du Canada. Statistiques canadiennes du cancer 2006.

26. Lemon S, Zapka J, Luckmann R, ChasanTaber L. Colorectal cancer screening participation: comparisons with mammography and prostate-specific antigen screening. Am J Pub Health. 2001;91(8):1264-1272.

27. Cokkinides VE, Chao A, Smith RA, Vernon SW, Thun MJ. Correlates of underutilization of colorectal cancer screening among U.S. adults, age 50 years and older. Prev Med. 2003;36(1):85-91.

28. Nadel MR, Blackman DK, Shapiro JA, Seeff LC. Are people being screened for colorectal cancer as recommended? Results from the National Health Interview Survey. Prev Med. 2002;35(3):199-206.

29. Walsh JME, Posner SF, Perez-Stable J. Colon cancer screening in the ambulatory setting. Prev Med. 2002;35(3):209-218.

30. Thompson B, Coronado GD, Solomon CC, McClerran DF, Neuhouser ML, Feng Z. Cancer prevention behaviours and socioeconomic status among Hispanics and nonHispanic whites in a rural population in the United States. Cancer Causes and Control. 2002;13(8):719-728 .

31. Brawarsky P, Brooks DR, Mucci LA. Correlates of colorectal cancer testing in Massachusetts men and women. Prev Med. 2003;36(6):659-668.

32. Ioannou GN, Chapko MK, Dominitz JA. Predictors of colorectal cancer screening participation in the United States. Am J Gastroenterol. 2003;98(9):2082-2091.

33. Madlensky L, Esplen MJ, Gallinger S, McLaughlin JR, Goel V. Relatives of colorectal cancer patients. Factors associated with screening behavior. Am J Prev Med. 2003; 25(3):187-194.

34. Harewood GC, Lieberman DA. Colonoscopy practice patterns since introduction of Medicare coverage for average-risk screening. Clin Gastroenterol Hepatol. 2004;2(1):72-77.

35. Flanagan WM, LePetit C, Berthelot JM, White KJ, Coombs BA, Jones-McLean E. Potential impact of population-based colorectal cancer screening in Canada. Chronic Dis Can. 2003; 24(4):81-88.

36. Colorectal cancer test use among persons aged $>$ or $=50$ years - United States, 2001. MMWR Morb Mortal Wkly Rep. 2003; 52(10):193-196.

37. Swan J, Breen N, Coates RJ, Rimer BK, Lee NC. Progress in cancer screening practices in the United States. Results from the 2000 National Health Interview Survey. Cancer. 2003; $97(6): 1528-1540$. 
38. Robertson RH, Burkhardt JH, Powell MP, Eloubeidi MA, Pisu M, Weissman NW. Trends in colon cancer screening procedures in the US Medicare and Tricare populations: 1999-2001. Prev Med 2006; 42(6):460-462.

39. Meissnner $\mathrm{HI}$, Breen $\mathrm{N}$, Klabunde $\mathrm{CN}$, Vernon SW. Patterns of colorectal cancer screening uptake among men and women in the United States. Cancer Epidemiology, Biomarkers \& Prevention 2006;15(2):389-394.

40. Miedema BB, Tatemichi S. Breast and cervical cancer screening for women between 50 and 69 years of age: what prompts women to screen? Womens Health Issues. 2003; 13(5):180-184.

41. Wadden N, Doyle GP. Breast cancer screening in Canada: a review. Can Assoc Radiol J. 2005;56(5):271-275.

42. Baier M, Calonge N, Cutter G et al. Validity of self-reported colorectal cancer screening behavior. Cancer Epidemiology, Biomarkers \& Prevention. 2000;9(2):229-232.

43. Madlensky L, McLaughlin J, Goel V. A comparison of self-reported colorectal screening with medical records. Cancer Epidemiology, Biomarkers \& Prevention. 2003;12(7):656-659.

44. Statistique Canada. Population de 1 an et plus selon les groupes d'âge, par mobilité (lieu de résidence 1 an auparavant), Canada, provinces et territoires, recensement de 1996 - Données-échantillon (20 \%). URL : http://www.statcan.ca/francais/census96/ apr14/mob2_f.htm . 